\title{
COMPARAÇÃO DE MÉTODOS CONVENCIONAL E ALTERNATIVO PARA DETERMINAR Cu, Fe, Mn E Zn EM EXTRATOS DE SOLOS OBTIDOS COM SOLUÇÃO DE MEHLICH-1 ${ }^{(1)}$
}

\author{
Ricardo Soares $^{(2)}$, Viviane Escaleira ${ }^{(3)}$, David Vilas Boas de Campos $^{(4)}$, Maria Inês Couto \\ Monteiro $^{(5)}$, Ricardo Erthal Santelli ${ }^{(6)}$ \& Alberto Carlos de Campos Bernardi ${ }^{(7)}$
}

\begin{abstract}
RESUMO
Nos laboratórios brasileiros, a determinação de micronutrientes para estudos de fertilidade de solos é realizada por extração com solução de Mehlich-1 (M-1), utilizando-se uma razão solo: extrator de 1:5 m/v e posterior quantificação por espectrometria de absorção atômica (AAS). No entanto, muitos laboratórios também empregam razão solo:extrator $M-1$ de 1:10 m/v para determinar macronutrientes e, ou, quantificar por espectrometria de emissão óptica com plasma indutivamente acoplado (ICP OES), em substituição ao AAS. Como estudos comparativos entre as concentrações de micronutrientes obtidos por esses métodos alternativos são escassos, o objetivo deste trabalho foi investigar se essas diferentes condições experimentais gerariam diferentes resultados. Os resultados foram estatisticamente tratados e revelaram que as concentrações de Fe e Mn, utilizandose ambas as razões de solo:extrator $\mathrm{M}-1$ (1:5 e 1:10 m/v) e de $\mathrm{Zn}$, na razão solo:extrator M-1 de 1:10 m/v, foram estatisticamente iguais, enquanto as concentrações de $\mathrm{Cu}$ em ambas as razões solo:extrator M-1 e de $\mathrm{Zn}$ na razão solo:extrator de 1:5 m/v foram diferentes, quando as duas técnicas de quantificação (ICP OES e AAS) foram comparadas. Também, todas as concentrações de $\mathrm{Cu}, \mathrm{Fe}$, $\mathrm{Mn}$ e $\mathrm{Zn}$ obtidas utilizando razão solo:extrator $M-1$ de 1:5 m/v foram diferentes daquelas encontradas com a razão de 1:10 m/v, independentemente da técnica de quantificação. A maioria dessas concentrações foi maior quando se usou a razão de 1:5 m/v e alterou a classe de
\end{abstract}

\footnotetext{
(1) Recebido para publicação em 5 de dezembro de 2011 e aprovado em 5 de março de 2013.

${ }^{(2)}$ Analista Ambiental, Instituto Estadual do Ambiente - INEA. Rua Sacadura Cabral 103, CEP 20081-261, Saúde, Rio de Janeiro (RJ). E-mail: ricardosoaresuff@yahoo.com.br

(3) Analista, Embrapa Solos. Rio de Janeiro (RJ). E-mail: viviane.escaleira@embrapa.br

(4) Pesquisador, Embrapa Solos. Rio de Janeiro (RJ). E-mail: david.campos@embrapa.br

(5) Pesquisador, Centro de Tecnologia Mineral - CETEM. Rio de Janeiro (RJ). E-mail: mcarneiro@cetem.gov.br

(6) Professor do Departamento de Química Analítica, Universidade Federal do Rio de Janeiro - UFRJ. Bolsista do CNPq. Rio de Janeiro (RJ). E-mail: santelli@iq.ufrj.br

(7) Pesquisador, Embrapa Pecuária Sudeste. Bolsista do CNPq. Rod. Washington Luiz, km 234, CEP 13560-970 São Carlos (SP). E-mail: alberto.bernardi@embrapa.br
} 
interpretação dos teores. Portanto, não recomenda-se o uso da razão solo:extrator de 1:5 para as extrações dos micronutrientes $\mathrm{Cu}$, Fe, Mn e $\mathrm{Zn}$ com solução M-1 de amostras de solo.

Termos de indexação: análise de solo, teste de identidade, validação analítica.

\author{
SUMMARY: COMPARISON OF CONVENTIONAL AND ALTERNATIVE \\ DETERMINATION METHODS FOR COPPER, IRON, \\ MANGANESE AND ZINC IN MEHLICH-1 EXTRACTED SOIL \\ SOLUTION
}

\begin{abstract}
In Brazilian laboratories, the determination of micronutrient contents for soil fertility analyses is based on extraction with Mehlich-1 solution (M-1), at a 1:5 w/v soil:extractant ratio, with subsequent quantification by atomic absorption spectrometry (AAS). However, many laboratories also use a 1:10 $\mathrm{w} / \mathrm{v}$ soil:extractant $M-1$ ratio for the determination of macronutrients, and/or quantification by optical emission spectrometry with inductively coupled plasma (ICP OES), instead of AAS. As comparative studies between the micronutrient concentrations obtained by these alternative methods are scarce, the aim of this work was to investigate significant differences in results from these different extraction and quantification methods. In this work, statistical analysis showed that the Fe and Mn concentrations using both soil:extractant $M-1$ ratios (1:5 and 1:10) and $Z n$ concentrations at 1:10 w/v soil: extractant $M-1$ ratio were similar regardless the quantification equipment. Howeve, the $C u$ concentrations using both soil:extractant $M-1$ ratios and the $Z n$ concentrations at 1:5 $\mathrm{w} / \mathrm{v}$ soil: extractant $M-1$ ratio differend according to the equipment used to quantifiy these nutrients. Also, all $\mathrm{Cu}$, $F e, M n$ and $Z n$ concentrations determined at 1:5 $\mathrm{w} / \mathrm{v}$ differed from those at 1:10 $\mathrm{w} / \mathrm{v}$ soil: extractant $M-1$ ratio, regardless of the quantification technique. Most of these concentrations were higher at a ratio of 1:5 $\mathrm{w} / \mathrm{v}$ and affected the classification of the contents. It is therefore not recommended to use a soil: extractant ratio of 1:5 for the extraction of the micronutrients $\mathrm{Cu}$, $\mathrm{Fe}, \mathrm{Mn}$ and $\mathrm{Zn}$ from soil samples.
\end{abstract}

Index terms: soil analyses, identity test, analytical validation.

\section{INTRODUÇÃO}

Em estudos de fertilidade de solos, a solução extratora Mehlich-1 (M-1) $\left(\mathrm{H}_{2} \mathrm{SO}_{4} 0,0125 \mathrm{~mol} \mathrm{~L}^{-1}+\right.$ $\mathrm{HCl} 0,05 \mathrm{~mol} \mathrm{~L}^{-1}$ ) tem sido empregada pela maioria dos laboratórios brasileiros para determinar a disponibilidade de macro e micronutrientes, como $\mathrm{Cu}$, Fe, Mn e Zn, para as plantas (Bernardi et al., 2002; Bortolon et al., 2009; Bortolon \& Gianello, 2010; Soares et al., 2012). O valor do $\mathrm{pH}$ (entre 2 e 3 ) dessa solução solubiliza os compostos contendo os macros e micronutrientes, enquanto $\mathrm{Cl}^{-}$restringe a readsorção dos fosfatos recém-extraídos (Mehlich, 1953; Silva et al., 1998). Para os micronutrientes, a relação recomendada de solo:extrator é de 1:5, enquanto para os macronutrientes, de 1:10 m/v (Mehlich, 1953; Silva et al., 1998). Entretanto, muitos laboratórios utilizam a razão solo:extrator M-1 de 1:10 ou de 1:5 m/v, tanto para especificar macro como micronutrientes (Bernardi et al., 2002).

Na maioria dos laboratórios de análises de solos, as concentrações de $\mathrm{Cu}, \mathrm{Fe}, \mathrm{Mn}$ e $\mathrm{Zn}$ nos extratos são definidas por espectrofotometria de absorção atômica (AAS) (Bernardi et al., 2002; Milagres et al., 2007; Bortolon \& Gianello, 2010). Nessa caracterização, é utilizada uma chama de ar/acetileno, que pode atingir temperaturas da ordem de $2.450 \mathrm{~K}$ (Soares et al., 2012; Lajunen, 1992). Essa temperatura é menor que aquela utilizada pela espectrometria de emissão óptica com plasma indutivamente acoplado (ICP OES) (10.000 K), que pode ser um método alternativo de determinação elementar nos extratos de solos por apresentar vantagens como maior sensibilidade, repetibilidade e reprodutibilidade (Soares et al., 2012).

Na última década, a técnica de ICP OES tem sido amplamente implementada em instituições de pesquisa e laboratórios privados para a determinação de analitos em diferentes extratos de solos e plantas (Bernardi et al., 2002; Soares et al., 2010, 2012). Entretanto, poucos trabalhos sobre a especificação de micronutrientes em extratos de solos obtidos com M1 por ICP OES são encontrados na literatura. Milagres et al. (2007) determinaram $\mathrm{Cu}, \mathrm{Fe}, \mathrm{Mn}$ e $\mathrm{Zn}$ nesses extratos por AAS e ICP OES e concluíram que os resultados por ambas as técnicas foram precisos e reprodutíveis, mas diferiram estatisticamente, comprometendo a interpretação dos resultados gerados por ICP OES, com base nos níveis críticos gerados por AAS. Bortolon \& Gianello (2010) compararam os resultados de $\mathrm{Cu}$ e $\mathrm{Zn}$ em extratos de solos obtidos 
com M-1, utilizando ICP OES e AAS. Os autores observaram que os resultados por ICP OES foram, na média, $35 \%$ maiores que aqueles encontrados por AAS e atribuíram esse fato à maior energia do plasma em comparação à da chama, que pode desprender $\mathrm{Cu} \mathrm{e}$ Zn, em solução, complexado com moléculas orgânicas. Em face disso, esses autores recomendaram a utilização de um fator de conversão para determinar o índice de disponibilidade de $\mathrm{Cu}$ e Zn no solo, quando determinados por ICP OES.

Como ainda são escassos os trabalhos realizados para testar a eficiência do ICP OES para a determinação de nutrientes em extratos de solos brasileiros (Milagres et al., 2007; Bortolon\&Gianello, 2010; Soares et al., 2010, 2012), mais estudos fazemse necessários.

Em face do exposto, este trabalho teve como objetivo comparar as concentrações de $\mathrm{Cu}, \mathrm{Fe}, \mathrm{Mn}$ e $\mathrm{Zn}$, em extratos de solos brasileiros, obtidas por método convencional (razão solo:extrator M-1 de 1:5 m/v e determinação por AAS) com aquelas encontradas utilizando ICP OES e razões solo:extrator M-1 de 1:5 e $1: 10 \mathrm{~m} / \mathrm{v}$, bem com usando AAS e razão solo:extrator de $1: 10 \mathrm{~m} / \mathrm{v}$.

\section{MATERIAL E MÉTODOS}

Foram avaliadas 15 amostras de solo utilizadas no "Programa de Análise da Qualidade de Laboratórios de Fertilidade" (PAQLF). Nesse programa, as amostras foram analisadas em triplicata por 82 laboratórios participantes (Bernardi et al., 2002). Utilizaram-se os métodos descritos no manual da Embrapa (Silva et al., 1998). No quadro 1, são apresentados os resultados fornecidos pelo PAQLF: valores médios, intervalos de confiança e desviospadrão dos resultados de $\mathrm{Cu}, \mathrm{Fe}$, Mn e Zn e algumas características dos solos: granulometria, $\mathrm{pH}$ e capacidade de troca catiônica (CTC).

Neste trabalho, o procedimento de extração de $\mathrm{Cu}$, $\mathrm{Fe}, \mathrm{Mn}$ e $\mathrm{Zn}$ foi realizado em triplicata e idêntico àquele descrito por Silva et al. (1998), que utilizam razão solo:extrator de 1:5 m/v, agitação (120 rpm) de $10 \mathrm{~cm}^{3}$ da amostra de solo com $50 \mathrm{~mL}$ de M-1 ( HCl $0,05 \mathrm{~mol} \mathrm{~L}^{-1}+\mathrm{H}_{2} \mathrm{SO}_{4} 0,0125 \mathrm{~mol} \mathrm{~L}^{-1}$ ), por $5 \mathrm{~min}$, e imediata filtração com papel-filtro da marca Whatman $\mathrm{n}^{\circ} 42 \mathrm{com} 0.45 \mathrm{~mm}$ de diâmetro do poro. Após essa etapa, aproximadamente $25 \mathrm{~mL}$ do filtrado foram transferidos para um recipiente de polietileno para determinar $\mathrm{Cu}, \mathrm{Fe}, \mathrm{Mn}$ e $\mathrm{Zn}$. Esse procedimento também foi realizado utilizando-se razão solo:extrator de $1: 10 \mathrm{~m} / \mathrm{v}$ (100 $\mathrm{mL}$ de M-1).

Nos extratos, os elementos $\mathrm{Cu}, \mathrm{Fe}, \mathrm{Mn}$ e $\mathrm{Zn}$ foram determinados por um AAS marca Perkin-Elmer, modelo AAnalyst 100 e software Winlab. Os comprimentos de onda, intensidade de corrente da lâmpada, energia e largura da fenda, utilizados para cada um dos elementos em estudo, foram: $324,8 \mathrm{~nm}$; $15 \mathrm{~mA} ; 73 \mathrm{~J}$; e $0,70 \mathrm{~mm}$, para o $\mathrm{Cu} ; 248,3 \mathrm{~nm} ; 15 \mathrm{~mA}$; $60 \mathrm{~J} ;$ e $0,20 \mathrm{~mm}$, para o Fe; $279,5 \mathrm{~nm} ; 20 \mathrm{~mA} ; 63 \mathrm{~J}$; e $0,20 \mathrm{~mm}$, para o $\mathrm{Mn}$; e $213,9 \mathrm{~nm} ; 15 \mathrm{~mA} ; 57 \mathrm{~J} ;$ e 0,70 $\mathrm{mm}$, para o $\mathrm{Zn}$, respectivamente.

Adicionalmente, os elementos $\mathrm{Cu}, \mathrm{Fe}, \mathrm{Mn}$ e $\mathrm{Zn}$ foram determinados por um ICP OES da marca Perkin-Elmer, modelo OPTIMA 3000 e software ICPWinlab. As condições de trabalho do equipamento foram: $1.500 \mathrm{~W}$ de potência, $15 \mathrm{~L} \mathrm{~min}^{-1}$ de vazão de gás de plasma, $0,5 \mathrm{~L} \mathrm{~min}^{-1}$ de vazão de gás auxiliar, $0,80 \mathrm{~L} \mathrm{~min}^{-1}$ para nebulização, vazão de purga do gás normal, resolução normal, em triplicata, tempo automático de medida do sinal ( 1 a $10 \mathrm{~s}$ ) e $2 \mathrm{~mL} \mathrm{~min}^{-1}$ de vazão da amostra. Os comprimentos de onda e alturas das janelas de observação utilizadas para cada um dos elementos em estudo foram: $324,759 \mathrm{~nm}$ e $8,0 \mathrm{~mm}$, para $\mathrm{Cu} ; 238,204 \mathrm{~nm}$ e $9,0 \mathrm{~mm}$, para Fe; $257,611 \mathrm{~nm}$ e 9,0 mm, para $\mathrm{Mn}$; e 213,857 $\mathrm{nm}$ e $9 \mathrm{~mm}$, para $\mathrm{Zn}$, respectivamente.

Os limites de detecção (LD) e de quantificação (LQ) foram calculados pelas equações $L D=3 \mathrm{~s} / \mathrm{b}$ e $\mathrm{LQ}=10$ s/b, em que (s) é o desvio-padrão de 10 determinações do branco e (b), a inclinação da curva de calibração (Ribeiro et al., 2008).

Foram selecionadas três amostras de solo (amostras 11, 12 e 14) que apresentaram grande amplitude nos resultados do PAQLF para avaliação da reprodutibilidade, enquanto a precisão, que diz respeito à repetibilidade, foi avaliada em todas as amostras analisadas. A reprodutibilidade, repetibilidade e comparação dos resultados, utilizando um esquema fatorial 4 x 15 , disposto em blocos casualizados completos, em triplicata, foram determinadas com procedimentos idênticos àqueles apresentados anteriormente para estudo de macronutrientes (Soares et al., 2012).

\section{RESULTADOS E DISCUSSÃo}

\section{Limites de detecção (LD) e de quantificação (LQ)}

Os LD e LQ obtidos por ICP OES para Fe e Mn foram 15 vezes menores, enquanto para $\mathrm{Cu}$ e $\mathrm{Zn}$ foram aproximadamente oito e seis vezes menores do que aqueles obtidos por AAS, respectivamente (Quadro 2). Portanto, ICP OES evidenciou ser uma técnica mais sensível (Ribeiro et al., 2008) do que AAS. Esses resultados estão de acordo com a capacidade do ICP OES em determinar baixas concentrações de diferentes analitos (Nolte, 2003; Soares et al., 2010, 2012). Em estudos anteriores utilizando essas mesmas amostras, Soares et al. (2010) verificaram que os LD e LQ para $\mathrm{Ca}, \mathrm{Mg}$ e $\mathrm{Al}$ em extratos de $\mathrm{KCl} 1 \mathrm{~mol} \mathrm{~L}^{-1}$, determinados por ICP OES, foram inferiores àqueles obtidos por titrimetria. Além disso, foram obtidos menores LD e LQ para $\mathrm{Na}, \mathrm{K}$ e $\mathrm{P}$ em extratos de M-1 quantificados 
Quadro 1. Análise granulométrica, pH, capacidade de troca catiônica (CTC), intervalo de confiança (IC), média das concentrações e desvios-padrão ( $\sigma$ ) para $\mathrm{Cu}, \mathrm{Fe}, \mathrm{Mn}$ e Zn obtidos pelo Programa de Análise da Qualidade de Laboratórios de Fertilidade (PAQLF)(1)

\begin{tabular}{|c|c|c|c|c|c|c|c|c|c|c|c|c|c|c|c|c|c|}
\hline \multirow[t]{3}{*}{ Solo } & \multirow[t]{3}{*}{ Argila } & \multirow{3}{*}{$\frac{\text { Silte }}{\text { g kg }^{-1}}$} & \multirow{3}{*}{ Areia } & \multirow[t]{3}{*}{$\mathbf{p H}\left(\mathrm{H}_{2} \mathrm{O}\right)$} & \multirow{3}{*}{$\frac{\mathrm{CTC}}{\mathrm{cmol}_{\mathrm{c}} \mathrm{dm}^{-3}}$} & \multicolumn{3}{|c|}{$\mathbf{C u}$} & \multicolumn{3}{|c|}{$\mathbf{F e}$} & \multicolumn{3}{|c|}{ Mn } & \multicolumn{3}{|c|}{$\mathbf{Z n}$} \\
\hline & & & & & & & & & & & & $-\mathbf{m g} \mathbf{d m}^{-3}$ & & & & & \\
\hline & & & & & & IC & Média & $\sigma$ & IC & Média & $\sigma$ & IC & Média & $\sigma$ & IC & Média & $\sigma$ \\
\hline 1 & 100 & 76 & 824 & 4,5 & 4,7 & $0,2-0,6$ & 0,38 & 0,13 & $60-146$ & 103,0 & 28,9 & $3,1-7,1$ & 5,15 & 1,00 & $1,1-2,1$ & 1,62 & 0,25 \\
\hline 2 & 360 & 120 & 520 & 5,2 & 7,2 & $0,2-0,6$ & 0,37 & 0,13 & $24-71$ & 47,7 & 23,6 & $28,0-86,5$ & 57,28 & 19,49 & $1,8-3,7$ & 2,76 & 0,62 \\
\hline 3 & 480 & 316 & 204 & 5,6 & 21,8 & $2,2-5,0$ & 3,61 & 0,95 & $46-136$ & 91,1 & 29,9 & $125-241$ & 183,0 & 29,13 & $12,5-28,5$ & 20,49 & 3,99 \\
\hline 4 & 340 & 106 & 554 & 6,5 & 9,3 & $0,2-0,7$ & 0,49 & 0,17 & $34-85$ & 59,6 & 25,8 & $75,2-183,2$ & 129,2 & 36,02 & $6,9-13,4$ & 10,15 & 1,64 \\
\hline 5 & 180 & 132 & 688 & 4,6 & 7,7 & $0,2-0,8$ & 0,29 & 0,46 & $67-193$ & 130,1 & 41,8 & $1,5-4,3$ & 2,92 & 0,93 & $1,7-3,2$ & 2,41 & 0,50 \\
\hline 6 & 344 & 140 & 516 & 4,5 & 9,5 & $0,5-1,2$ & 0,85 & 0,24 & $46-102$ & 73,9 & 18,5 & $4,9-10,7$ & 7,78 & 1,46 & $0,7-1,5$ & 1,10 & 0,20 \\
\hline 7 & 318 & 179 & 503 & 6,1 & 14,8 & $1,3-3,1$ & 2,18 & 0,43 & $52-171$ & 111,3 & 39,7 & $56,9-194,5$ & 125,7 & 45,85 & $5,4-9,5$ & 7,49 & 1,03 \\
\hline 8 & 527 & 223 & 250 & 4,7 & 12,3 & $0,1-0,5$ & 0,34 & 0,15 & $223-486$ & 354,5 & 87,4 & $12,6-26,0$ & 19,2 & 3,37 & $0,9-2,05$ & 1,49 & 0,29 \\
\hline 9 & 151 & 93 & 754 & 6,0 & 6,8 & $0,3-0,7$ & 0,47 & 0,13 & 27-68 & 47,6 & 13,9 & $29,8-61,0$ & 45,4 & 10,41 & $1,8-3,6$ & 2,70 & 0,47 \\
\hline 10 & 65 & 38 & 897 & 5,8 & 2,5 & $0,2-0,4$ & 0,33 & 0,08 & $34-72$ & 52,7 & 9,5 & $29,7-79,1$ & 54,3 & 16,48 & $2,6-4,6$ & 3,59 & 0,52 \\
\hline 11 & 247 & 118 & 635 & 5,0 & 7,1 & $2,6-4,6$ & 3,57 & 0,51 & $55-116$ & 85,7 & 15,1 & $8,9-20,6$ & 14,7 & 2,94 & $2,0-2,0$ & 2,00 & 0,00 \\
\hline 12 & 227 & 190 & 583 & 5,6 & 11,6 & $0,3-0,7$ & 0,52 & 0,15 & $47-131$ & 88,8 & 28,1 & $105-291$ & 198 & 61,77 & $6,3-12,7$ & 9,50 & 2,16 \\
\hline 13 & 241 & 118 & 641 & 5,1 & 7,6 & $0,2-0,7$ & 0,48 & 0,15 & $142-289$ & 215,3 & 49,0 & $65,3-133$ & 99,1 & 22,56 & $12,3-23,2$ & 17,77 & 2,74 \\
\hline 14 & 188 & 89 & 723 & 5,2 & 4,9 & $0,6-1,5$ & 1,04 & 0,45 & 27-61 & 44,3 & 11,3 & $11,1-21$ & 16,1 & 3,3 & $1,6-3,2$ & 2,40 & 0,50 \\
\hline 15 & 740 & 97 & 163 & 3,7 & 14,1 & $0,1-0,7$ & 0,27 & 0,45 & $109-261$ & 184,9 & 50,9 & $3,7-7,4$ & 5,52 & 1,24 & $0,2-1,4$ & 0,81 & 0,59 \\
\hline
\end{tabular}

${ }^{(1)}$ Métodos Embrapa (Silva et al., 1998).

Quadro 2. Limites de detecção (LD) e de quantificação (LQ) para Cu, Fe, Mn e Zn determinados por AAS e ICP OES

\begin{tabular}{|c|c|c|c|c|}
\hline Técnica & $\mathbf{C u}$ & $\mathbf{F e}$ & Mn & Zn \\
\hline & \multicolumn{4}{|c|}{$\mathrm{mg} \mathrm{dm} \mathrm{m}^{-3}$} \\
\hline & \multicolumn{4}{|c|}{$\mathrm{LD}$} \\
\hline AAS & 0,3 & 0,3 & 0,3 & 0,3 \\
\hline ICP OES & 0,03 & 0,02 & 0,02 & 0,04 \\
\hline & \multicolumn{4}{|c|}{ LQ } \\
\hline AAS & 0,8 & 0,9 & 0,9 & 0,8 \\
\hline ICP OES & 0,09 & 0,06 & 0,06 & 0,12 \\
\hline
\end{tabular}

por ICP OES, quando comparados a métodos espectroscópicos convencionais (Soares et al., 2012).

\section{Precisão e reprodutibilidade}

Para que os resultados sejam considerados precisos e reprodutíveis, o desvio-padrão relativo (DPR - \%) dentro de uma série de determinações (precisão) não deve ser maior que $10 \%$, enquanto os DPR (\%) entre as diferentes séries (reprodutibilidade) não devem ultrapassar a $20 \%$ (Klesta \& Bartz, 1996; Milagres et al., 2007). No quadro 3, são apresentados os valores médios das concentrações de $\mathrm{Cu}, \mathrm{Fe}, \mathrm{Mn}$ e $\mathrm{Zn}$ e os respectivos DPR (\%) obtidos no estudo de repetibilidade.

As concentrações de $\mathrm{Cu}$ variaram de abaixo do $\mathrm{LD}$ $\left(<0,3 \mathrm{mg} \mathrm{dm}^{-3}\right)$ a $5,53 \mathrm{mg} \mathrm{dm}{ }^{-3}$. Foram obtidas concentrações de $\mathrm{Cu}$ menores que o $\mathrm{LD}$ somente quando foi utilizado AAS. Esse fato ocorreu em $47 \%$ das amostras (1, 2, 4, 5, 7, 8 e 10), quando a razão solo:extrator foi de 1:5; e em $60 \%(1,2,4,5,7,8,9$, 10 e 13), quando a razão solo:extrator foi de $1: 10$, indicando que provavelmente a maior diluição do extrato impediu a detecção do analito, pois trata-se de técnica menos sensível que ICP OES. Foram obtidos resultados não precisos em $80 \%$ das amostras, quando foram utilizados ICP OES e razão solo:extrator de 1:10 $\mathrm{m} / \mathrm{v}$; em $40 \%$, quando usaram-se AAS e razão solo:extrator de 1:5 m/v; em $33 \%$, quando utilizaramse AAS e razão solo:extrator de $1: 10 \mathrm{~m} / \mathrm{v}$; e em $13 \%$, quando empregaram-se ICP OES e razão solo:extrator de $1: 5 \mathrm{~m} / \mathrm{v}$, indicando que essa última condição apresentou maior precisão.

As concentrações de Fe variaram de 20,4 a 232 $\mathrm{mg} \mathrm{dm}{ }^{-3}$. Resultados não precisos foram obtidos somente quando se utilizaram razão solo:extrator de $1: 10 \mathrm{~m} / \mathrm{v}$ e AAS (amostras 6, 11 e 14) e ICP OES (amostras 2, 7 e 11), em $20 \%$ das amostras.

As concentrações de Mn variaram de 2,52 a 243 $\mathrm{mg} \mathrm{dm}{ }^{-3}$. Somente duas amostras apresentaram precisões insatisfatórias: amostra 11 , em todas as condições testadas, e amostra 15 , utilizando razão solo:extrator de $1: 5 \mathrm{~m} / \mathrm{v}$ e ambas as técnicas analíticas, indicando que a extração foi provavelmente comprometida pela forma do analito associada a essas amostras de solo.

As concentrações de $Z n$ variaram de abaixo do $L D$ $\left(<0,3 \mathrm{mg} \mathrm{dm}^{-3}\right)$ a $17,5 \mathrm{mg} \mathrm{dm}^{-3}$. Concentrações de $\mathrm{Zn}$ abaixo do $\mathrm{LD}$ foram conseguidas em $27 \%$ das amostras $(6,7,8$ e 15) somente quando foram utilizadas AAS e 
Quadro 3. Teores de Cu, Fe, Mn e Zn com os seus respectivos desvios-padrão relativos - DPR, entre parênteses, obtidos por AAS e ICP OES (n = 3)

\begin{tabular}{|c|c|c|c|c|c|c|c|c|c|c|c|c|c|c|c|c|}
\hline \multirow{3}{*}{ Solo } & \multicolumn{4}{|c|}{$\mathbf{C u}$} & \multicolumn{4}{|c|}{$\mathbf{F e}$} & \multicolumn{4}{|c|}{ Mn } & \multicolumn{4}{|c|}{$\mathbf{Z n}$} \\
\hline & \multicolumn{2}{|c|}{ AAS } & \multicolumn{2}{|c|}{ ICP OES } & \multicolumn{2}{|c|}{ AAS } & \multicolumn{2}{|c|}{ ICP OES } & \multicolumn{2}{|c|}{ AAS } & \multicolumn{2}{|c|}{ ICP OES } & \multicolumn{2}{|c|}{ AAS } & \multicolumn{2}{|c|}{ ICP OES } \\
\hline & $1: 5$ & 1:10 & 1:5 & $1: 10$ & 1:5 & $1: 10$ & 1:5 & $1: 10$ & 1:5 & 1:10 & 1:5 & 1:10 & 1:5 & $1: 10$ & $1: 5$ & $1: 10$ \\
\hline & & & & & & & & $\mathrm{mg} \mathrm{dm} \mathrm{d}^{-3}(\%$ & & & & & & & & \\
\hline 1 & $<\mathrm{LD}$ & $<\mathrm{LD}$ & $0,24(9)$ & $0,22(28)$ & $96,0(2)$ & $94,2(4)$ & $96,0(3)$ & $92,0(5)$ & $5,12(4)$ & $3,74(6)$ & $4,90(2)$ & $3,59(10)$ & $1,50(5)$ & $1,16(5)$ & $1,53(4)$ & $1,20(4)$ \\
\hline 2 & $<\mathrm{LD}$ & $<\mathrm{LD}$ & $0,23(8)$ & $0,19(35)$ & $45,9(5)$ & $33,7(10)$ & $48,1(3)$ & $34,3(13)$ & $59,7(7)$ & $45,9(4)$ & $61,7(10)$ & $47,7(6)$ & $2,13(6)$ & $1,0(2)$ & $2,01(4)$ & $0,99(6)$ \\
\hline 3 & $2,09(1)$ & $2,01(19)$ & $2,12(2)$ & $1,94(12)$ & $53,9(3)$ & $46,6(1)$ & $54,9(2)$ & $44,7(4)$ & $242(5)$ & $222(2)$ & $243(4)$ & $214(1)$ & $15,1(7)$ & $13,2(6)$ & $13,6(6)$ & $13,3(6)$ \\
\hline 4 & $<\mathrm{LD}$ & $<\mathrm{LD}$ & $0,23(6)$ & $0,18(18)$ & $93,0(4)$ & $86,3(10)$ & $93,9(3)$ & $83,6(6)$ & $144(3)$ & $153(5)$ & $153(10)$ & $128(3)$ & $8,65(2)$ & $7,07(19)$ & $8,77(2)$ & $7,90(17)$ \\
\hline 5 & $<\mathrm{LD}$ & $<\mathrm{LD}$ & $0,20(3)$ & $0,16(42)$ & $78,4(2)$ & $78,7(2)$ & $75,9(5)$ & $75,3(7)$ & $3,31(9)$ & $2,80(4)$ & $3,03(7)$ & $2,52(1)$ & $2,51(3)$ & $2,19(5)$ & $2,35(4)$ & $2,18(21)$ \\
\hline 6 & $0,43(13)$ & $0,32(12)$ & $0,57(2)$ & $0,54(11)$ & $44,7(6)$ & $41,3(12)$ & $45,7(6)$ & $41,5(7)$ & $7,76(10)$ & $6,39(2)$ & $7,17(8)$ & $6,27(2)$ & $0,66(11)$ & $<\mathrm{LD}$ & $0,74(11)$ & $0,47(20)$ \\
\hline 7 & $<\mathrm{LD}$ & $<\mathrm{LD}$ & $1,93(8)$ & $1,67(18)$ & $158(3)$ & $159(3)$ & $141(6)$ & $145(11)$ & $120(1)$ & $121(2)$ & $117(6)$ & $119(5)$ & $7,54(12)$ & $<\mathrm{LD}$ & $7,34(1)$ & $7,58(6)$ \\
\hline 8 & $<\mathrm{LD}$ & $<\mathrm{LD}$ & $0,18(12)$ & $0,15(16)$ & $230(3)$ & $201,5(3)$ & $231(3)$ & $211(8)$ & $17,3(1)$ & $16,4(2)$ & $17,4(1)$ & $14,3(2)$ & $1,00(17)$ & $<\mathrm{LD}$ & $0,88(5)$ & $0,73(7)$ \\
\hline 9 & $0,36(15)$ & $<\mathrm{LD}$ & $0,30(4)$ & $0,34(17)$ & $35,5(3)$ & $22,2(10)$ & $36,5(2)$ & $20,4(9)$ & $36,3(5)$ & $19,4(5)$ & $38,58(4)$ & $20,7(7)$ & $2,05(6)$ & $0,98(1)$ & $2,03(1)$ & $1,14(2)$ \\
\hline 10 & $<\mathrm{LD}$ & $<\mathrm{LD}$ & $0,21(4)$ & $0,19(6)$ & $44,0(3)$ & $31,9(9)$ & $45,6(5)$ & $34,9(7)$ & $62,6(6)$ & $41,9(4)$ & $60,4(8)$ & $38,4(4)$ & $2,66(7)$ & $1,45(1)$ & $3,18(7)$ & $1,65(8)$ \\
\hline 11 & $4,79(4)$ & $5,53(1)$ & $3,53(16)$ & $3,57(14)$ & $62,7(5)$ & $34,1(17)$ & $63,4(6)$ & $38,1(15)$ & $9,71(14)$ & $9,28(12)$ & $8,12(13)$ & $8,04(10)$ & $5,80(4)$ & $4,08(3)$ & $5,18(2)$ & $3,76(17)$ \\
\hline 12 & $0,59(14)$ & $0,64(11)$ & $0,46(8)$ & $0,66(10)$ & $52,7(2)$ & $46,3(4)$ & $53,7(1)$ & $45,4(5)$ & $183(6)$ & $179(8)$ & $183(3)$ & $182(8)$ & $6,59(3)$ & $6,38(19)$ & $6,70(1)$ & $6,76(20)$ \\
\hline 13 & $0,35(19)$ & $<\mathrm{LD}$ & $0,29(2)$ & $0,28(20)$ & $232(1)$ & $232(2)$ & $218(3)$ & $228(5)$ & $83,6(9)$ & $72,5(6)$ & $84,7(6)$ & $69,3(1)$ & $17,5(1)$ & $15,8(11)$ & $16,2(7)$ & $15,4(11)$ \\
\hline 14 & $0,53(12)$ & $0,62(11)$ & $0,62(9)$ & $0,51(15)$ & $34,3(9)$ & $27,6(22)$ & $37,5(7)$ & $27,6(8)$ & $14,7(2)$ & $11,2(8)$ & $15,7(10)$ & $9,96(8)$ & $2,12(13)$ & $1,48(11)$ & $2,14(5)$ & $1,53(12)$ \\
\hline 15 & $0,65(14)$ & $0,51(13)$ & $0,84(1)$ & $0,77(6)$ & $160(3)$ & $141(7)$ & $160(3)$ & $143(7)$ & $4,75(18)$ & $2,91(7)$ & $4,97(20)$ & $2,70(9)$ & $0,47(14)$ & $<\mathrm{LD}$ & $0,44(7)$ & $0,32(7)$ \\
\hline
\end{tabular}


razão solo:extrator de $1: 10 \mathrm{~m} / \mathrm{v}$, indicando que provavelmente a maior diluição do extrato impediu a detecção do analito. Obtiveram-se resultados não precisos em $47 \%$ das amostras, quando foram utilizados ICP OES e razão solo:extrator de 1:10 m/v; em $27 \%$, quando usaram-se AAS e razão solo:extrator de 1:10 m/ v; em $33 \%$, quando empregaram-se AAS e razão solo:extrator de $1: 5 \mathrm{~m} / \mathrm{v}$; e em $7 \%$, quando utilizaramse ICP OES e razão solo:extrator de $1: 5 \mathrm{~m} / \mathrm{v}$, indicando que essa última condição apresentou maior precisão. Esses resultados foram semelhantes àqueles encontrados por Milagres et al. (2007), os quais também detectaram maior precisão do ICP OES, comparativamente ao AAS na determinação das concentrações de Cu e Zn extraídos com M-1, razão solo:extrator de 1:5 m/v, bem como as precisões para $\mathrm{Cu}$ e $\mathrm{Zn}$, presentes em baixas concentrações, foram seriamente comprometidas quando determinadas por AAS.

Os resultados do estudo de reprodutibilidade realizado para as amostras 11, 12 e 14 encontram-se no quadro 4 . Foram obtidas boas reprodutibilidades nas determinações de $\mathrm{Cu}, \mathrm{Fe}, \mathrm{Mn}$ e Zn, independentemente da técnica de quantificação ou da razão solo:extrator. Os resultados de reprodutibilidade para Fe e $\mathrm{Mn}$, utilizando razão solo:extrator M-1 de 1:5 m/v, concordaram com aqueles obtidos por Milagres et al. (2007). Entretanto, para $\mathrm{Cu}$ eZn, presentes em menores concentrações, esses autores observaram que o uso de ICP OES contribuiu para obter melhores reprodutibilidades.

\section{Comparação dos resultados analíticos}

As concentrações de $\mathrm{Cu}, \mathrm{Fe}, \mathrm{Mn}$ e Zn obtidas pelos diferentes métodos foram comparadas por um teste de identidade proposto por Leite \& Oliveira (2002) (Quadro 5). As 20 determinações que apresentaram resultados abaixo do $\mathrm{LD}$ (Quadro 3) foram descartadas para o não comprometimento da avaliação estatística. Observaram-se altas correlações entre as concentrações para Fe e Mn (razão solo:extrator de
1:5 ou $1: 10 \mathrm{~m} / \mathrm{v}$ ) e Zn (razão solo:extrator de $1: 10 \mathrm{~m} / \mathrm{v}$ ) quantificadas por ICP OES, quando comparadas com aquelas encontradas por AAS. Ao contrário, para $\mathrm{Cu}$ (razão solo:extrator de 1:5 ou 1:10 m/v) e Zn (razão solo:extrator de 1:5 m/v) não foram encontradas altas correlações, indicando que os resultados foram diferentes. Essas concentrações de $\mathrm{Cu}(1,4$ a $29 \%)$ e Zn $(0,9$ a $20 \%)$ obtidas por ICP OES foram maiores em 50 e $40 \%$ das amostras, quando comparadas com aquelas obtidas por AAS, respectivamente. Também, Milagres et al. (2007) e Bortolon \& Gianelho (2010) obtiveram concentrações diferentes para $\mathrm{Cu}$ e $\mathrm{Zn}$ quantificados por ICP OES e AAS.

No quadro 5, é apresentada a comparação dos resultados obtidos com razão solo:extrator de 1:10 m/ $\mathrm{v}$ com aqueles encontrados com razão solo:extrator de $1: 5 \mathrm{~m} / \mathrm{v}$, utilizando o teste de identidade. Foram observadas diferenças significativas para todas as concentrações de $\mathrm{Cu}, \mathrm{Fe}, \mathrm{Mn}$ e $\mathrm{Zn}$, utilizando ambas as técnicas de quantificação. A maioria dos resultados obtidos com razão solo:extrator de $1: 10 \mathrm{~m} / \mathrm{v}$ foi menor que aqueles conseguidos com razão solo:extrator 1:5 $\mathrm{m} / \mathrm{v}$. Exceção ocorreu para determinação de $\mathrm{Cu}$ por AAS (amostras 11, 12 e 14) e ICP OES (amostras 11 e 12 ); Fe por AAS (amostras 5, 7 e 13) e ICP OES (amostras 7 e 13); Mn por AAS (amostras 4 e 7) e ICP OES (amostra 7) e Zn por AAS (amostras 6, 7, 8 e 15) e ICP OES (amostras 7 e 12).

As diferenças observadas nos teores alteraram a classe de interpretação dos teores dos nutrientes, conforme proposta por Alvarez et al. (1999). As alterações dessas classes foram mais frequentes para 0 Zn (amostras 2, 6, 7, 8, 9, 10 e 14), Fe (amostras 2, 6, $9,10,11$ e 14) e em menor escala para o $\mathrm{Cu}$ (amostras $1,2,4,5,7,10$ e 13) e Mn (amostra 14). Em todas as amostras que se observaram discrepâncias na classificação das faixas de teores, verificou-se que a relação 1:5 superestimou a extração desses nutrientes. Portanto, os maiores teores de micronutrientes obtidos

Quadro 4. Média e reprodutibilidade dos métodos para determinação de Cu, Fe, Mn e Zn por AAS e ICP OES $(\mathbf{n}=3)$

\begin{tabular}{|c|c|c|c|c|c|c|c|c|c|c|c|c|c|c|c|c|}
\hline \multirow[b]{3}{*}{ Razão solo:extrator } & \multicolumn{4}{|c|}{$\mathbf{C u}$} & \multicolumn{4}{|c|}{$\mathbf{F e}$} & \multicolumn{4}{|c|}{ Mn } & \multicolumn{4}{|c|}{$\mathbf{Z n}$} \\
\hline & \multicolumn{2}{|c|}{ AAS } & \multicolumn{2}{|c|}{ ICP OES } & \multicolumn{2}{|c|}{ AAS } & \multicolumn{2}{|c|}{ ICP OES } & \multicolumn{2}{|c|}{ AAS } & \multicolumn{2}{|c|}{ ICP OES } & \multicolumn{2}{|c|}{ AAS } & \multicolumn{2}{|c|}{ ICP OES } \\
\hline & 1:5 & 1:10 & $1: 5$ & 1:10 & 1:5 & 1:10 & 1:5 & 1:10 & 1:5 & 1:10 & $1: 5$ & 1:10 & $1: 5$ & 1:10 & $1: 5$ & $1: 10$ \\
\hline \multicolumn{17}{|l|}{ Solo 11} \\
\hline Média (mg dm ${ }^{-3}$ ) & 4,6 & 5,6 & 3,9 & 3,7 & 63,8 & 37,2 & 65,7 & 40,3 & 10,1 & 8,8 & 9,0 & 8,0 & 6,1 & 4,2 & 5,4 & 4,0 \\
\hline Reprodutibilidade (\%) & 5 & 7 & 17 & 19 & 6 & 15 & 7 & 17 & 10 & 15 & 12 & 15 & 6 & 16 & 5 & 14 \\
\hline \multicolumn{17}{|l|}{ Solo 12} \\
\hline Média (mg dm ${ }^{-3}$ ) & 0,7 & 0,7 & 0,5 & 0,7 & 53,7 & 47,1 & 54,2 & 46,3 & 183 & 181 & 179 & 6,9 & 6,6 & 6,9 & 7,2 & 6,4 \\
\hline Reprodutibilidade (\%) & 12 & 14 & 6 & 8 & 6 & 7 & 4 & 6 & 10 & 11 & 4 & 7 & 7 & 12 & 3 & 10 \\
\hline \multicolumn{17}{|l|}{ Solo 14} \\
\hline Média $\left(\mathrm{mg} \mathrm{dm}^{-3}\right)$ & 0,6 & 0,6 & 0,7 & 0,6 & 35,1 & 28,3 & 38,3 & 26,5 & 14,1 & 11,7 & 16,7 & 12,4 & 2,6 & 1,6 & 2,8 & 1,5 \\
\hline Reprodutibilidade (\%) & 12 & 15 & 10 & 12 & 12 & 17 & 9 & 10 & 13 & 14 & 8 & 11 & 10 & 13 & 7 & 10 \\
\hline
\end{tabular}

Reprodutibilidade: desvio-padrão relativo, em \%, dos resultados entre as séries analíticas. 
Quadro 5. Resultados obtidos pela aplicação do teste de identidade para comparação de AAS e ICP OES e razões de solo:extrator 1:5 e 1:10

\begin{tabular}{|c|c|c|c|c|c|c|c|c|c|}
\hline Elemento & $\mathbf{n}^{(\mathbf{1})}$ & $\mathbf{B}_{0}^{(2)}$ & $\mathbf{B}_{1}^{(3)}$ & $\mathbf{r}^{(4)}$ & $\overline{\boldsymbol{e}}^{(\mathbf{5})}$ & $\mathbf{F}\left(\mathbf{H}_{0}\right)^{(6)}$ & $t \bar{e}^{(7)}$ & $\mathbf{r} \geq(1-|\bar{e}|)$ & Conclusão \\
\hline \multicolumn{10}{|c|}{ AAS x ICP OES (Razão 1:5) } \\
\hline $\mathrm{Cu}$ & 24 & $-0,015^{*}$ & $1,138^{*}$ & 0,997 & 0,127 & $36,24 *$ & $0,744^{\mathrm{ns}}$ & Sim & $\mathrm{AAS} \neq \mathrm{ICP}$ OES \\
\hline $\mathrm{Fe}$ & 45 & $1,378^{\mathrm{ns}}$ & $0,997^{\mathrm{ns}}$ & 0,999 & 0,021 & $22,79^{\text {ns }}$ & $0,936^{\mathrm{ns}}$ & Sim & $\mathrm{AAS}=\mathrm{ICP}$ OES \\
\hline $\mathrm{Mn}$ & 45 & $0,329^{\text {ns }}$ & $1,007^{\mathrm{ns}}$ & 0,998 & 0,002 & $0,816^{\mathrm{ns}}$ & $0,959^{\mathrm{ns}}$ & Sim & $\mathrm{AAS}=\mathrm{ICP}$ OES \\
\hline $\mathrm{Zn}$ & 45 & $0,217^{*}$ & $0,915^{\mathrm{ns}}$ & 0,995 & $-0,014$ & $21,15^{*}$ & $0,836^{\text {ns }}$ & Sim & AAS $\neq$ ICP OES \\
\hline \multicolumn{10}{|c|}{ AAS x ICP OES (Razão 1:10) } \\
\hline $\mathrm{Cu}$ & 18 & $0,164^{*}$ & $0,942^{*}$ & 0,994 & 0,241 & $6,91 *$ & $0,817^{\mathrm{ns}}$ & Sim & $\mathrm{AAS} \neq \mathrm{ICP}$ OES \\
\hline $\mathrm{Fe}$ & 45 & $-1,842^{\text {ns }}$ & $1,042^{\text {ns }}$ & 0,993 & 0,017 & $3,09^{\text {ns }}$ & $0,909^{\text {ns }}$ & Sim & $\mathrm{AAS}=\mathrm{ICP}$ OES \\
\hline $\mathrm{Mn}$ & 45 & $-0,385^{\mathrm{ns}}$ & $1,004^{\mathrm{ns}}$ & 0,999 & $-0,028$ & $0,289^{\mathrm{ns}}$ & $0,993^{\mathrm{ns}}$ & Sim & $\mathrm{AAS}=\mathrm{ICP}$ OES \\
\hline $\mathrm{Zn}$ & 33 & $0,290^{\mathrm{ns}}$ & $0,961^{\mathrm{ns}}$ & 0,966 & 0,054 & $0,44^{\mathrm{ns}}$ & $0,939^{\text {ns }}$ & Sim & $\mathrm{AAS}=\mathrm{ICP}$ OES \\
\hline \multicolumn{10}{|c|}{ RAZÃO 1:5 x RAZÃO 1:10 (AAS) } \\
\hline $\mathrm{Cu}$ & 18 & $0,113^{*}$ & $0,738^{*}$ & 0,977 & $-0,146$ & $31,54^{*}$ & $0,553^{\text {ns }}$ & Sim & Razão 1:5 f Razão 1:10 \\
\hline $\mathrm{Fe}$ & 45 & $-5,982^{*}$ & $0,935^{\mathrm{ns}}$ & 0,991 & $-0,162$ & $47,94^{*}$ & $0,382^{\text {ns }}$ & Sim & Razão 1:5 = Razão 1:10 \\
\hline $\mathrm{Mn}$ & 45 & $-3,843^{*}$ & $0,933^{*}$ & 0,992 & $-0,188$ & $25,76^{*}$ & $0,583^{\mathrm{ns}}$ & Sim & Razão 1:5 f Razão 1:10 \\
\hline $\mathrm{Zn}$ & 45 & $-0,617^{*}$ & $0,924 *$ & 0,985 & $-0,262$ & $27,22^{*}$ & $0,408^{\text {ns }}$ & Sim & Razão 1:5 f Razão 1:10 \\
\hline \multicolumn{10}{|c|}{ RAZÃO 1:5 x RAZÃO 1:10 (ICP OES) } \\
\hline $\mathrm{Cu}$ & 45 & $-0,016^{*}$ & $0,943^{\text {ns }}$ & 0,983 & $-0,105$ & $7,62 *$ & $0,678^{\mathrm{ns}}$ & Sim & Razão 1:5 f Razão 1:10 \\
\hline $\mathrm{Fe}$ & 45 & $-9,869^{*}$ & $0,982^{\text {ns }}$ & 0,988 & $-0,169$ & $28,91^{*}$ & $0,410^{*}$ & Sim & Razão 1:5 f Razão 1:10 \\
\hline $\mathrm{Mn}$ & 45 & $-3,965^{*}$ & $0,917^{\mathrm{ns}}$ & 0,989 & $-0,213$ & $29,35^{*}$ & $0,528 *$ & Sim & Razão 1:5 f Razão 1:10 \\
\hline $\mathrm{Zn}$ & 45 & $-0,519 *$ & $0,985^{\mathrm{ns}}$ & 0,985 & $-0,237$ & $11,15^{*}$ & $0,560 *$ & Sim & Razão 1:5 $\neq$ Razão 1:10 \\
\hline
\end{tabular}

${ }^{(1)}$ Número de observações; ${ }^{(2)}$ Intercepto da regressão; ${ }^{(3)}$ Coeficiente da regressão linear; ${ }^{(4)}$ Coeficiente de correlação linear simples; ${ }^{(5)}$ Erro médio; ${ }^{(6)}$ Teste F; ${ }^{(7)}$ Teste t para o erro médio; ${ }^{\text {ns }} \mathrm{e} *$ : não-significativo e significativo a $5 \%$, respectivamente.

na relação $1: 5$ podem induzir à inadequada interpretação dos teores e, consequentemente, ocasionar erros no manejo da fertilidade do solo, uma vez que poderiam indicar que a adubação não era necessária ou a dose era abaixo da ideal.

Deve-se ter em mente que o teste de identidade utilizado é um procedimento estatístico e objetivo para verificar a existência de diferenças significativas entre dois métodos (Leite \& Oliveira, 2002) e que seu uso e suas interpretações devem ser criteriosos. Portanto, a existência de diferenças significativas encontradas entre as concentrações de $\mathrm{Cu}$ (razões solo:extrator de 1:5 e $1: 10 \mathrm{~m} / \mathrm{v}$ ) e Zn (razão solo:extrator de 1:5 m/v), usando ICP OES e AAS, não inviabiliza o uso do ICP OES, pois essa técnica apresenta muitas vantagens como: maior sensibilidade, repetibilidade e reprodutibilidade.

\section{CONCLUSÕES}

1. A técnica de ICP OES foi mais sensível, precisa e reprodutível para determinar $\mathrm{Cu}, \mathrm{Fe}, \mathrm{Mn}$ e $\mathrm{Zn}$ do que a de AAS.

2. Ouso da razão solo:extrator M-1 de 1:10 impossibilitou detectar $\mathrm{Cu}$ e $\mathrm{Zn}$ por AAS, quando em concentrações menores que o $\mathrm{LD}$ do método $\left(0,3 \mathrm{mg} \mathrm{dm}^{-3}\right)$.
3. Os resultados de Fe e Mn obtidos por ICP OES foram estatisticamente semelhantes àqueles determinados por AAS. A maior capacidade do ICP OES em determinar baixas concentrações de $\mathrm{Cu}$ e $\mathrm{Zn}$ gerou resultados estatisticamente diferentes aos obtidos por AAS, mas isso não inviabiliza o seu uso.

4. Os resultados de $\mathrm{Cu}, \mathrm{Fe}, \mathrm{Mn}$ e $\mathrm{Zn}$ obtidos com a razão solo:extrator de 1:10 foram estatisticamente diferentes daqueles encontrados utilizando-se a razão solo:extrator de 1:5. A maior parte dos teores de nutrientes detectados com razão solo:extrator de 1:5 $\mathrm{m} / \mathrm{v}$ foi maior que aqueles obtidos com a razão solo:extrator $1: 10 \mathrm{~m} / \mathrm{v}$, alterando a classe de interpretação dos resultados.

5.0 uso da razão solo:extrator de 1:5 para as extrações dos micronutrientes $\mathrm{Cu}, \mathrm{Fe}, \mathrm{Mn}$ e $\mathrm{Zn}$ com solução M-1 em amostras de solo é questionável, de acordo com este trabalho.

\section{AGRADECIMENTOS}

À Fundação Carlos Chagas Filho de Amparo à Pesquisa do Estado do Rio de Janeiro (FAPERJ) e ao CNPq pela concessão de bolsas. Ao Dr. Hélio Garcia Leite, por gentilmente ter cedido uma planilha de confecção do teste de identidade. 


\section{LITERATURA CITADA}

ALVAREZV., V.H.; NOVAES, R.F.; BARROS, N.F.; CANTARUTTI, R.B.\& LOPES, A.S. Interpretação dos resultados das análises de solos. In: RIBEIRO, A.C.; GUIMARAES, P.T.G.\& ALVAREZ V., V.H.,eds. Recomendação para o uso de corretivos e fertilizantes em Minas Gerais: 5. Aproximação. Viçosa, MG, Comissão de Fertilidade do Solo do Estado de Minas Gerais, 1999. p.25-32.

BERNARDI, A.C.C.; SILVA, C.A.; PÉREZ, D.V. \& MENEGUELLI, N.A. Analytical quality program of soil fertility laboratories that adopt Embrapa methods in Brazil. Commun. Soil Sci. Plant Anal., 33:2661-2672, 2002.

BORTOLON, L.; GIANELLO, C. \& SCHLINDWEIN, J.A. Avaliação da disponibilidade de fósforo no solo para o milho pelos métodos Mehlich-1 e Mehlich-3. Sci. Agríc 10:305-312, 2009 .

BORTOLON, L. \& GIANELLO, C. Simultaneous multielement extraction with the Mehlich-1 solution for Southern Brazilian soils determined by ICP-OES and the effects on the nutrients recommendations to crops. R. Bras. Ci. Solo, 34:125-132, 2010.

KLESTA, E.J. \& BARTZ, J.K. Quality assurance and control. In: BARTELS, J.M. \& BIGHAM, J.M., eds. Methods of soil analysis. Chemical methods. Madison, Soil Science Society of America, 1996. Part 3. 248p.

LAJUNEN, L.H.J. Spectrochemical analysis by atomic absorption and emission. London, Royal Society of Chemistry, 1992. 241p.

LEITE, H.G. \& OLIVEIRA, F.H.T. Statistical procedure to test identity between analytical methods. Commun. Soil Sci. Plant Anal., 37:1105-1118, 2002.
MEHLICH, A. Determination of $\mathrm{P}, \mathrm{Ca}, \mathrm{Mg}, \mathrm{K}, \mathrm{Na}$ and $\mathrm{NH}_{4}$ by North Carolina soil testing laboratories. Raleigh, University of North Carolina, 1953. 8p.

MILAGRES, J.J.M.; ALAVAREZ V., V.H.; CANTARUTTI, R.B. \& NEVES, J.C.L.Determinação de $\mathrm{Fe}, \mathrm{Zn}, \mathrm{Cu}$ e Mn extraídos do solo por diferentes extratores e dosados por espectrofotometria de emissão ótica em plasma induzido e espectrofotometria de absorção atômica. R. Bras. Ci. Solo,31:237-245, 2007.

NOLTE, J. ICP: Emission spectrometry: A pratical guide. Weinheim, Willey-VCH, 2003. 267p.

RIBEIRO, F.A.L.; FERREIRA, M.M.C.; MORANO, S.C.; SILVA, L.R. \& SCHNEIDER, R.P. Planilha de validação: uma nova ferramenta para estimar figuras de mérito na validação de métodos analíticos univariados. Quím. Nova, 31:164-171, 2008.

SILVA, F.C.; EIRA, P.A.; BARRETO, W.O.; PÉREZ, D.V. \& SILVA, C.A. Manual de métodos de análises químicas para avaliação da fertilidade do solo. Rio de Janeiro, Embrapa-CNPS, 1998. 56p. (Documentos, 3)

SOARES, R.; ESCALEIRA, V.; MONTEIRO, M.I.C.; PONTES, F.V.M.; SANTELLI, R.E. \& BERNARDI, A.C.C. Uso de ICP OES e titrimetria para a determinação de cálcio, magnésio e alumínio em amostras de solos. R. Bras. Ci. Solo,34:1553-1559, 2010.

SOARES, R.; ESCALEIRA, V.; MONTEIRO, M.I.C.; SANTELLI, R.E. \& BERNARDI, A.C.C. Comparação de métodos convencional e alternativo para determinação de sódio, potássio e fósforo em extratos de solos obtidos com solução Mehlich-1. R. Bras. Ci. Solo, 33:105-112, 2012. 